Supporting Information for:

\title{
Intramolecular C-H and N-H Transfer by Ruthenium(II) Amidophosphine Complexes
}

Michael D. Fryzuk*, Michael Petrella, and Brian O. Patrick

\author{
Department of Chemistry \\ University of British Columbia \\ 2036 Main Mall \\ Vancouver, BC, Canada \\ V6T 1Z1
}

Email: fryzuk@chem.ubc.ca

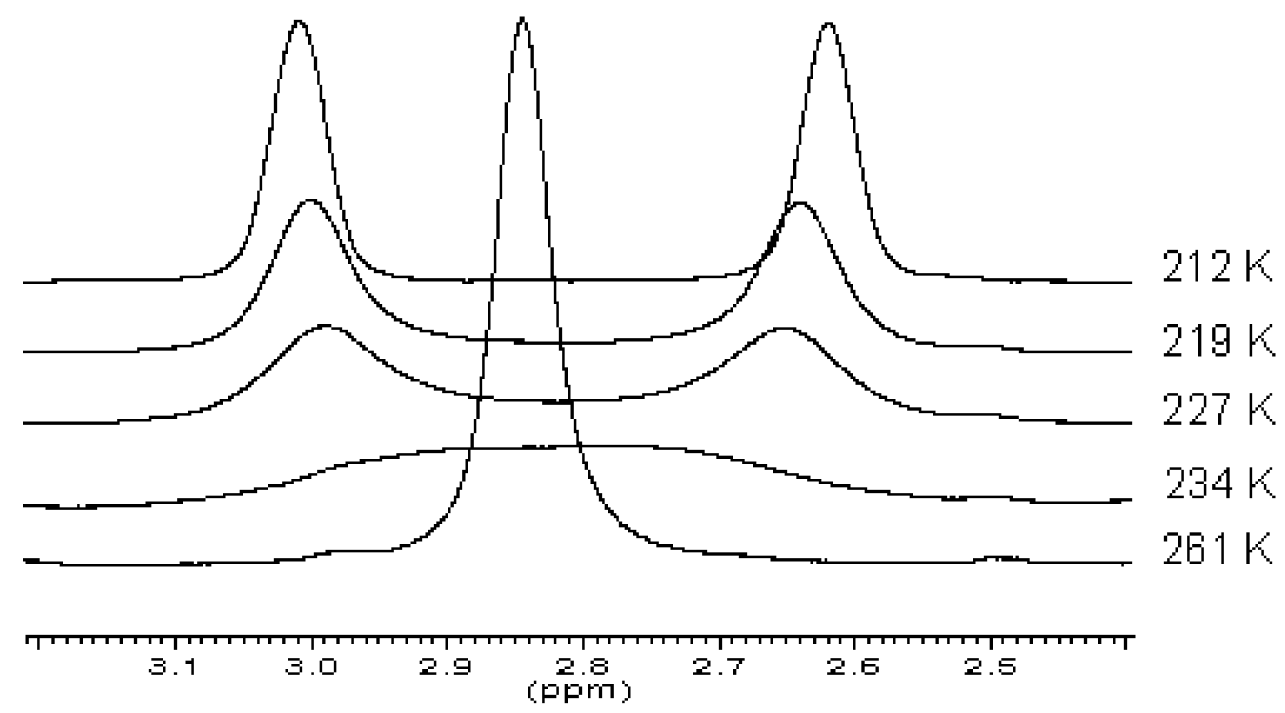

Figure S1. The effect of temperature on the methylene resonance of the cyclooctadiene ligand in the $500 \mathrm{MHz}{ }^{1} \mathrm{H}$ NMR spectrum of $\left[\mathrm{P}_{2} \mathrm{~N}_{2}\right] \mathrm{Ru}\left(\eta^{2}: \eta^{2}-\mathrm{C}_{8} \mathrm{H}_{12}\right)(\mathbf{1})$. 
Table S1. Calculated rate constants $(k)$ for the fluxionality of the $\left[\mathrm{P}_{2} \mathrm{~N}_{2}\right]$ ligand in the complex $\left[\mathrm{P}_{2} \mathrm{~N}_{2}\right] \mathrm{Ru}\left(\eta^{2}: \eta^{2}-\mathrm{C}_{8} \mathrm{H}_{12}\right)(\mathbf{1})$; some of these data are illustrated in Figure $\mathrm{S} 1$.

\begin{tabular}{c|c}
\hline Rate Constant, $\mathrm{k}\left(\mathrm{s}^{-1}\right)$ & Temperature, $\mathrm{T}(\mathrm{K})$ \\
\hline $5304 \pm 265$ & $243 \pm 1$ \\
$865 \pm 43$ & $234 \pm 1$ \\
$560 \pm 28$ & $230 \pm 1$ \\
$433 \pm 22$ & $227 \pm 1$ \\
$66 \pm 3$ & $219 \pm 1$ \\
$18 \pm 1$ & $212 \pm 1$ \\
\hline
\end{tabular}

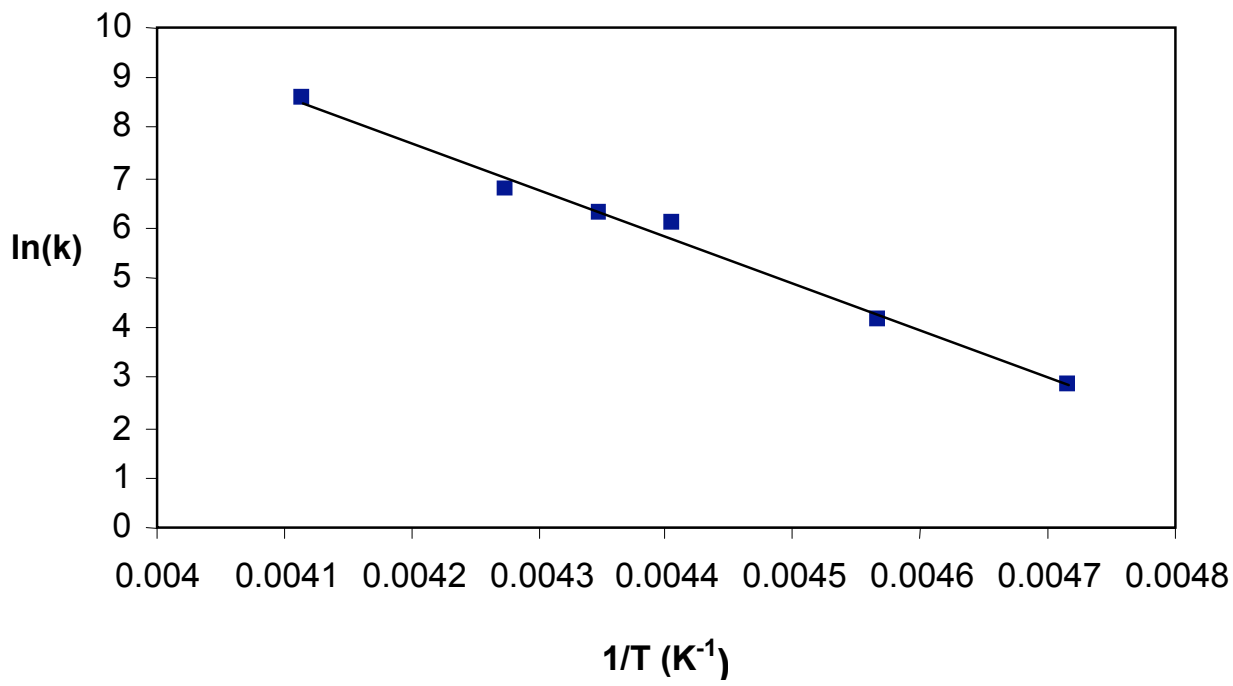

Figure S2. Arrhenius plot for the fluxionality of the $\left[\mathrm{P}_{2} \mathrm{~N}_{2}\right]$ ligand framework in the complex $\left[\mathrm{P}_{2} \mathrm{~N}_{2}\right] \mathrm{Ru}\left(\eta^{2}: \eta^{2}-\mathrm{C}_{8} \mathrm{H}_{12}\right)(\mathbf{1})\left(\mathrm{R}^{2}=0.9920\right.$ and $\left.\mathrm{E}_{\mathrm{a}}=18.6 \pm 1.6 \mathrm{kcal} \mathrm{mol}^{-1}\right)$ for the data in Table S1. 
Table S2. Calculated equilibrium constants $(K)$ for the equilibrium between diastereomers endo-3 and exo-3 in toluene- $d_{8}$.

\begin{tabular}{c|c}
\hline Equilibrium Constant, $K$ & Temperature, T (K) \\
\hline $0.46 \pm 0.04$ & $293 \pm 1$ \\
$0.43 \pm 0.04$ & $285 \pm 1$ \\
$0.39 \pm 0.04$ & $265 \pm 1$ \\
$0.34 \pm 0.04$ & $245 \pm 1$ \\
$0.28 \pm 0.03$ & $225 \pm 1$ \\
\hline
\end{tabular}

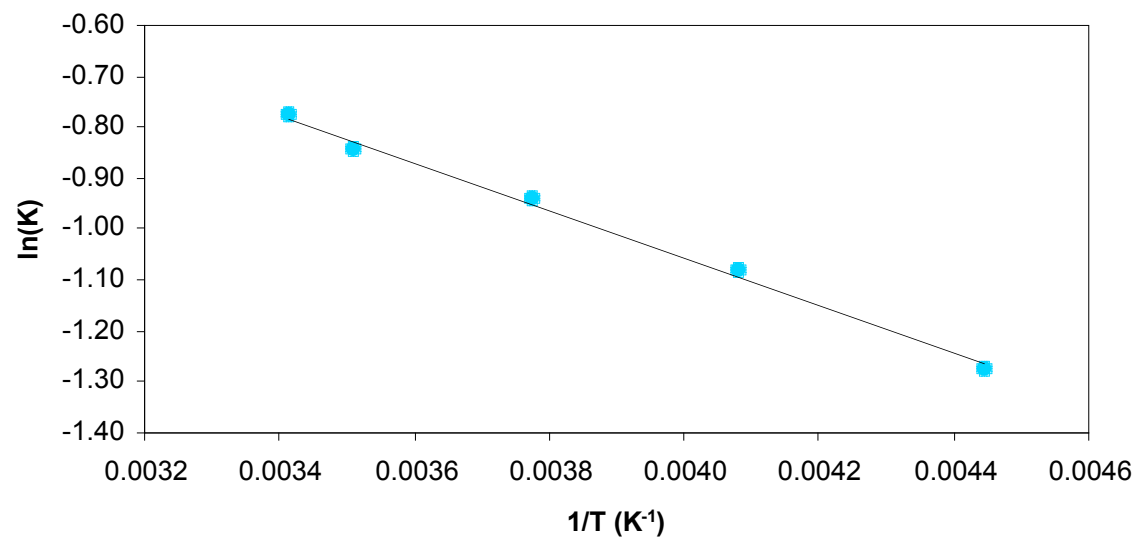

Figure S3. Van't Hoff plot for the equilibrium between diastereomers endo-3 and exo-3 $\left(R^{2}=0.9954\right)$ using the data in Table S2. 
Table S3. Calculated rate constants for the reversible intramolecular proton transfer responsible for the inter-conversion of diastereomers endo-3 and exo-3.

\begin{tabular}{c|c}
\hline Rate Constant, ${\mathrm{k}\left(\mathrm{s}^{-1}\right)}^{-1}$ & Temperature, $\mathrm{T}(\mathrm{K})$ \\
\hline $648 \pm 32$ & $320 \pm 1$ \\
$308 \pm 15$ & $308 \pm 1$ \\
$61 \pm 3$ & $293 \pm 1$ \\
$30 \pm 2$ & $285 \pm 1$ \\
\hline
\end{tabular}

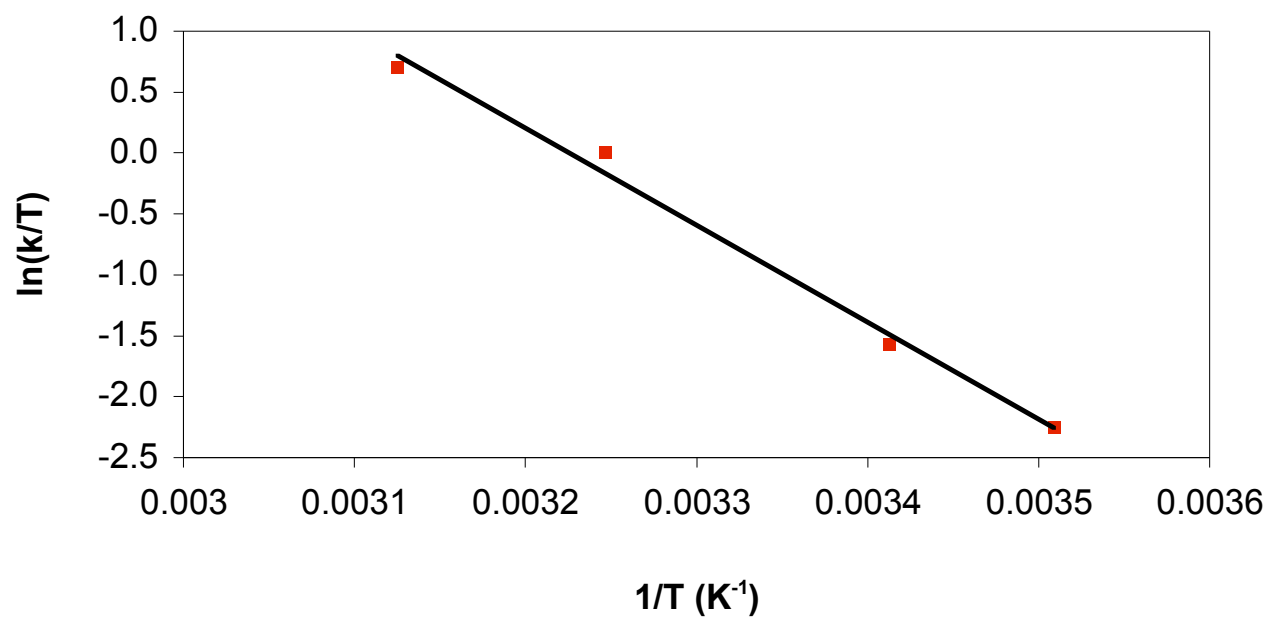

Figure S4. Eyring plot for the inter-conversion of diastereomers endo-3 and exo-3 $\quad\left(\mathrm{R}^{2}\right.$ $=0.9922$ ). Slope and intercept provide $\Delta H^{\ddagger}=16 \pm 1 \mathrm{kcal} \mathrm{mol}^{-1}$ and $\Delta S^{\ddagger}=4 \pm 4 \mathrm{eu}$, respectively, using the data in Table S3. 
Table S4. ${ }^{1} \mathrm{H}$ resonances of the cyclooctadienyl ligand for complexes 3-5 including $J_{\mathrm{HH}}$ coupling constants for complex exo-3 (determined from simulation data).

\begin{tabular}{ccccccc}
\hline & endo-3 & exo-3 & Li-4 & Na-4 & endo-5 & exo-5 \\
\hline $\mathrm{H}_{\mathrm{a}}$ & 4.23 & 4.68 & 2.20 & 2.17 & 4.25 & 4.50 \\
$\mathrm{H}_{\mathrm{b}}$ & 4.25 & 3.92 & 2.45 & 2.29 & 4.00 & 3.87 \\
$\mathrm{H}_{\mathrm{c}}$ & 2.36 & 1.83 & 1.60 & 1.18 & 2.10 & 2.08 \\
$\mathrm{H}_{\mathrm{c}}$, & 1.58 & 1.30 & 1.10 & 1.07 & 1.47 & 1.45 \\
$\mathrm{H}_{\mathrm{d}}$ & 2.18 & 2.20 & 1.70 & 1.76 & 2.25 & 2.12 \\
$\mathrm{H}_{\mathrm{d}}$ & 1.50 & 1.75 & 1.40 & 1.33 & 1.85 & 1.75 \\
$\mathrm{H}_{\mathrm{e}}$ & 4.20 & 3.50 & 3.40 & 3.10 & 4.16 & 3.60 \\
$\mathrm{H}_{\mathrm{f}}$ & 1.64 & 1.50 & 3.42 & 3.45 & 1.52 & 1.43 \\
$\mathrm{H}_{\mathrm{g}}$ & 2.85 & 3.37 & 4.30 & 4.35 & 2.90 & 3.27 \\
$\mathrm{H}_{\mathrm{h}}$ & 2.70 & 3.09 & 3.40 & 3.45 & 2.77 & 2.95 \\
$\mathrm{H}_{\mathrm{h}}$, & 2.00 & 2.15 & 2.50 & 2.50 & 2.05 & 2.20 \\
\hline
\end{tabular}

exo-3: ${ }^{3} J_{\mathrm{ab}}=7.80 \mathrm{~Hz},{ }^{3} J_{\mathrm{ah}}=7.70 \mathrm{~Hz},{ }^{3} J_{\mathrm{ah}}=7.30 \mathrm{~Hz},{ }^{3} J_{\mathrm{bc}}=6.20 \mathrm{~Hz},{ }^{3} J_{\mathrm{bc}}=6.10 \mathrm{~Hz}$, ${ }^{2} J_{\mathrm{cc}^{\prime}}=14.60 \mathrm{~Hz},{ }^{3} J_{\mathrm{cd}}=7.60 \mathrm{~Hz},{ }^{3} J_{\mathrm{cd}}=6.00 \mathrm{~Hz},{ }^{3} J_{\mathrm{c}^{\prime} \mathrm{d}}=7.20 \mathrm{~Hz},{ }^{3} J_{\mathrm{c}^{\prime} \mathrm{d}^{\prime}}=8.60 \mathrm{~Hz},{ }^{2} J_{\mathrm{dd}},=$ $17.0 \mathrm{~Hz},{ }^{3} J_{\text {de }}=5.60 \mathrm{~Hz},{ }^{3} J_{\text {d'e }}=5.80 \mathrm{~Hz},{ }^{3} J_{\text {ef }}=8.00 \mathrm{~Hz},{ }^{3} J_{\text {fg }}=9.90 \mathrm{~Hz},{ }^{3} J_{\text {gh }}=7.90 \mathrm{~Hz}$, ${ }^{3} J_{\mathrm{gh}},=4.20 \mathrm{~Hz},{ }^{2} J_{\mathrm{hh}}$, $=19.30 \mathrm{~Hz}$. (Determined using the simulation software in the NMR program Mestrix)

Table S5. ${ }^{13} \mathrm{C}$ resonances of the cyclooctadienyl ligand for complexes 3-5. ${ }^{2} J_{\mathrm{PC}}$ values $(\mathrm{Hz})$ are given in parenthesis.

\begin{tabular}{ccccccc}
\hline & endo-3 & exo-3 & $\mathbf{L i - 4}$ & $\mathbf{N a - 4}$ & endo-5 & exo-5 \\
\hline $\mathrm{C}_{1}$ & $61.4(6.5)$ & $65.3(5.8)$ & $32.6(6.5)$ & $33.2(6.4)$ & $61.6(6.3)$ & $62.9(5.9)$ \\
$\mathrm{C}_{2}$ & $108.1(10.5)$ & $110.0(11.3)$ & $67.8(5.5)$ & $68.2(5.8)$ & $107.6(10.6)$ & $108.1(11.6)$ \\
$\mathrm{C}_{3}$ & 30.3 & 27.0 & 29.8 & 27.9 & 28.5 & 26.9 \\
$\mathrm{C}_{4}$ & 32.8 & 36.3 & 30.4 & 27.7 & 35.0 & 36.9 \\
$\mathrm{C}_{5}$ & 61.6 & 64.9 & $46.2(2.2)$ & $46.0(2.1)$ & 61.8 & 63.7 \\
$\mathrm{C}_{6}$ & 71.6 & 70.8 & 100.5 & 100.5 & 70.1 & 69.9 \\
$\mathrm{C}_{7}$ & 35.6 & 36.5 & $44.3(18.9)$ & $45.0(19.2)$ & 35.6 & 36.2 \\
$\mathrm{C}_{8}$ & 25.2 & 26.6 & 22.3 & 22.7 & 25.5 & 26.5 \\
\hline
\end{tabular}




\section{Synthesis of $[\mathrm{Na}(\mathrm{THF})]\left([\mathrm{NPN}] \mathrm{Ru}\left(\eta^{3}: \eta^{2}-\mathrm{C}_{8} \mathrm{H}_{11}\right)\right)(5)$}

This sodium ruthenate complex 5 was prepared in a similar fashion as described for 4 in the main paper; in this case, the quantities were: $0.054 \mathrm{~g}(0.294 \mathrm{mmol})$ of $\mathrm{NaN}\left(\mathrm{SiMe}_{3}\right)_{2}$ and $0.189 \mathrm{~g}(0.294 \mathrm{mmol})$ of 3 were employed. Yield: $0.193 \mathrm{~g}, 89 \%$. Xray quality crystals were grown from a saturated toluene solution at $-40{ }^{\circ} \mathrm{C}$. ${ }^{1} \mathrm{H} \mathrm{NMR}$ $\left(\mathrm{C}_{6} \mathrm{D}_{6}, 298 \mathrm{~K}, 500 \mathrm{MHz}\right): \delta 0.10,0.20,0.35$ and 0.50 (s, $\mathrm{SiCH}_{3}, 12$ total), $\delta 1.00$ (m, AA'BX, PCHH, 1H), $1.07\left(\mathrm{~m}, \mathrm{CH}_{\mathrm{c}} H_{c}, 1 \mathrm{H}\right), \delta 1.14$ (m, overlapping, $\left.\mathrm{OCH}_{2} \mathrm{CH}_{2}, 2 \mathrm{H}\right), \delta$ 1.18 (m, overlapping, $\left.\mathrm{CH}_{c} \mathrm{H}_{\mathrm{c}^{\prime}}, 1 \mathrm{H}\right), \delta 1.33\left(\mathrm{~m}, \mathrm{CH}_{\mathrm{d}} H_{d}, 1 \mathrm{H}\right), \delta 1.44$ (m, AA'BX, PCHH, $1 \mathrm{H}), \delta 1.65$ (m, AA'BX, PCHH, 1H), $\delta 1.73$ (m, overlapping, AA'BX, PCH $H, 1 \mathrm{H}), \delta$ $1.76\left(\mathrm{~m}, \mathrm{CH}_{d} \mathrm{H}_{\mathrm{d}}, 1 \mathrm{H}\right), \delta 2.17\left(\mathrm{~m}, \mathrm{CH}_{a}, 1 \mathrm{H}\right), \delta 2.29\left(\mathrm{~m}, \mathrm{CH} H_{b}, 1 \mathrm{H}\right), \delta 2.50\left(\mathrm{~m}, \mathrm{CH} H_{h}, 1 \mathrm{H}\right)$, $\delta 3.06$ (m, overlapping, $\left.\mathrm{OCH}_{2} \mathrm{CH}_{2}, 2 \mathrm{H}\right), \delta 3.10$ (m, overlapping, $\left.\mathrm{CH}_{e}, 1 \mathrm{H}\right), \delta 3.45(\mathrm{~m}$, overlapping, $\left.\mathrm{CH}_{h} \mathrm{H}_{\mathrm{h}}, 1 \mathrm{H}\right), \delta 3.45$ (m, overlapping, $\left.\mathrm{CH}_{f}, 1 \mathrm{H}\right), \delta 4.35\left(\mathrm{~m}, \mathrm{C} H_{g}, 1 \mathrm{H}\right), \delta 6.58$

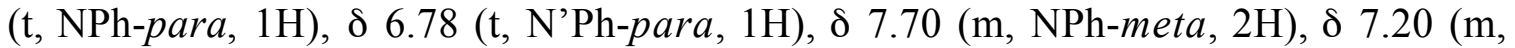
overlapping, NPh-ortho, 2H), $\delta 7.20$ (m, overlapping, PPh-para, 1H), $\delta 7.39$ (m, N'Phmeta, 2H), $\delta 8.05$ (m, AX, PPh-ortho, 2H), $\delta 8.30$ (d, N'Ph-ortho, $2 \mathrm{H}) . \quad{ }^{31} \mathrm{P}\left\{{ }^{1} \mathrm{H}\right\} \mathrm{NMR}$ $\left(\mathrm{C}_{6} \mathrm{D}_{6}, 298 \mathrm{~K}, 202.5 \mathrm{MHz}\right): \delta 46.8(\mathrm{~s}) .{ }^{13} \mathrm{C}\left\{{ }^{1} \mathrm{H}\right\} \mathrm{NMR}\left(\mathrm{C}_{7} \mathrm{D}_{8}, 298 \mathrm{~K}, 125.8 \mathrm{MHz}\right)$ selected data: $\delta 22.7\left(\mathrm{~s}, \mathrm{C}_{8}\right), \delta 27.7\left(\mathrm{~s}, \mathrm{C}_{4}\right), \delta 27.9\left(\mathrm{~s}, \mathrm{C}_{3}\right), \delta 33.2\left(\mathrm{~d}, \mathrm{C}_{1},{ }^{2} J_{\mathrm{PC}}=6.4 \mathrm{~Hz}\right), \delta$ $45.0\left(\mathrm{~d}, \mathrm{C}_{7},{ }^{2} J_{\mathrm{PC}}=19.2 \mathrm{~Hz}\right), \delta 46.0\left(\mathrm{~d}, \mathrm{C}_{5},{ }^{2} J_{\mathrm{PC}}=2.1 \mathrm{~Hz}\right), \delta 68.2\left(\mathrm{~d}, \mathrm{C}_{2},{ }^{2} J_{\mathrm{PC}}=5.8 \mathrm{~Hz}\right), \delta$ $100.5\left(\mathrm{~s}, \mathrm{C}_{6}\right)$.

\section{X-Ray Crystallography}

All measurements were made on an ADSC CCD area detector coupled with a Rigaku AFC7 diffractometer with graphite monochromated Mo-K $\alpha$ radiation. Data were collected at temperatures between $-75 \pm 1{ }^{\circ} \mathrm{C}$ and $-100 \pm 1{ }^{\circ} \mathrm{C}$ (see Table S6) and processed using the $\mathrm{d}^{*} \mathrm{TREK}$ program. ${ }^{1}$ The structures were solved by direct methods; $;^{2,3}$ the data were corrected for Lorentz and polarization effects. and expanded using Fourier techniques. ${ }^{4}$ With the exception of the $\mathrm{N}-H$ hydrogen atoms, which were refined isotropically, all other hydrogen atoms were included but not refined. Non-hydrogen atoms were refined anisotropically. 
Table S6. Crystallographic Data and Structure Refinement Data for complexes $\left[\mathrm{P}_{2} \mathrm{~N}_{2}\right] \mathrm{Ru}\left(\eta^{2}: \eta^{2}-\mathrm{C}_{8} \mathrm{H}_{12}\right)(\mathbf{1}),\left[\mathrm{P}_{2} \mathrm{NNH}\right] \mathrm{Ru}\left(\mathrm{C}_{6} \mathrm{H}_{4} \mathrm{PPh}_{2}\right)(2)$, endo-[NPNH$] \mathrm{Ru}\left(\eta^{3}: \eta^{2}-\mathrm{C}_{8} \mathrm{H}_{11}\right)$ $\left(\right.$ endo-3) and $[\mathrm{Li}(\mathrm{THF})]\left([\mathrm{NPN}] \mathrm{Ru}\left(\eta^{3}: \eta^{2}-\mathrm{C}_{8} \mathrm{H}_{11}\right)\right)(\mathbf{4})$.

\begin{tabular}{|c|c|c|c|c|}
\hline & 1 & 2 & endo-3 & 4 \\
\hline Formula & $\begin{array}{l}\mathrm{C}_{32} \mathrm{H}_{56} \mathrm{~N}_{2} \mathrm{P}_{2} \mathrm{Si}_{4} \cdot \mathrm{C} \\
{ }_{6} \mathrm{H}_{14} \mathrm{Ru}\end{array}$ & $\begin{array}{l}2\left[\mathrm{C}_{42} \mathrm{H}_{57} \mathrm{~N}_{2} \mathrm{P}_{3} \mathrm{Si}_{4}\right. \\
\mathrm{Ru}] . \mathrm{C}_{5} \mathrm{H}_{12}\end{array}$ & $\mathrm{C}_{32} \mathrm{H}_{43} \mathrm{~N}_{2} \mathrm{PRuSi}_{2}$ & $\begin{array}{l}\mathrm{C}_{36} \mathrm{H}_{50} \mathrm{~N}_{2} \mathrm{PSi}_{2} \mathrm{Li} \\
\mathrm{ORu} \cdot \mathrm{C}_{7} \mathrm{H}_{8}\end{array}$ \\
\hline $\mathrm{FW}$ & 830.34 & 1864.67 & 643.92 & 814.10 \\
\hline Colour, Habit & yellow, platelet & yellow, chip & red, prism & red, block \\
\hline Crystal size, mm & $\begin{array}{l}0.50 \times 0.20 \times \\
0.05\end{array}$ & $\begin{array}{l}0.25 \times 0.25 \times \\
0.10\end{array}$ & $\begin{array}{l}0.25 \times 0.15 \times \\
0.10\end{array}$ & $\begin{array}{llll}0.40 & \mathrm{x} & 0.30 & \mathrm{x} \\
0.10 & & & \end{array}$ \\
\hline Crystal system & monoclinic & orthorhombic & trigonal & orthorhombic \\
\hline Space group & $\mathrm{P} 2 / \mathrm{n}(\# 13)$ & Pbca (\#61) & R3 (\#148) & $\mathrm{P} 2{ }_{1} 2_{1} 2_{1}(\# 19)$ \\
\hline $\mathrm{a}, \AA$ & $11.113(1)$ & $20.3845(8)$ & $42.2752(7)$ & $9.0674(3)$ \\
\hline $\mathrm{b}, \AA$ & $10.854(1)$ & $21.7255(8)$ & & $19.9274(5)$ \\
\hline $\mathrm{c}, \AA$ & $17.994(2)$ & $43.150(2)$ & $9.0004(3)$ & $23.055(1)$ \\
\hline$\alpha, \operatorname{deg}$ & & & & \\
\hline$\beta, \operatorname{deg}$ & $96.944(3)$ & & & \\
\hline$\gamma, \operatorname{deg}$ & & & & \\
\hline $\mathrm{V}, \AA^{3}$ & $2154.6(3)$ & 19109(1) & $13930.4(4)$ & $4165.8(2)$ \\
\hline Z & 2 & 8 & 18 & 4 \\
\hline $\mathrm{T},{ }^{\circ} \mathrm{C}$ & $-85 \pm 1$ & $-100 \pm 1$ & $-100 \pm 1$ & $-75 \pm 1$ \\
\hline$\rho_{\text {calc }}, g / \mathrm{cm}^{3}$ & 1.280 & 1.296 & 1.382 & 1.298 \\
\hline $\mathrm{F}_{000}$ & 884.00 & 7824.00 & 6048.00 & 1712.00 \\
\hline$\mu(\mathrm{MoK} \alpha), \mathrm{cm}^{-1}$ & 5.77 & 5.61 & 6.60 & 5.07 \\
\hline correction factors & $0.6695-1.0000$ & $0.7109-1.0000$ & $0.7589-1.0000$ & $0.7804-1.0000$ \\
\hline $2 \theta_{\max }, \operatorname{deg}$ & 55.8 & 50.0 & 55.8 & 55.8 \\
\hline total no. of reflns & 20052 & 151583 & 36357 & 31090 \\
\hline no. of unique reflns & 4799 & 16723 & 6957 & 9060 \\
\hline $\mathrm{R}_{\text {int }}$ & 0.046 & 0.112 & 0.060 & 0.047 \\
\hline $\begin{array}{l}\text { no. observations (I } \\
>\operatorname{n\sigma } \sigma(\mathrm{I}))\end{array}$ & $3680(n=2)$ & $13461(n=2)$ & $4940(n=3)$ & $7849(\mathrm{n}=3)$ \\
\hline no. of variables & 218 & 1006 & 391 & 480 \\
\hline $\mathrm{R}\left(\mathrm{F}^{2}\right.$, all data $)$ & 0.047 & 0.083 & 0.050 & 0.046 \\
\hline $\mathrm{R}_{\mathrm{w}}\left(\mathrm{F}^{2}\right.$, all data $)$ & 0.095 & 0.158 & 0.076 & 0.069 \\
\hline $\mathrm{R}(\mathrm{F}, \mathrm{I}>\mathrm{n} \sigma(\mathrm{I}))$ & $0.035(n=2)$ & $0.064(n=2)$ & $0.028(n=3)$ & $0.026(n=3)$ \\
\hline $\mathrm{R}_{\mathrm{w}}(\mathrm{F}, \mathrm{I}>\mathrm{n \sigma}(\mathrm{I}))$ & $0.091(n=2)$ & $0.148(n=2)$ & $0.035(n=3)$ & $0.032(n=3)$ \\
\hline Gof & 1.00 & 1.10 & 0.88 & 0.94 \\
\hline
\end{tabular}




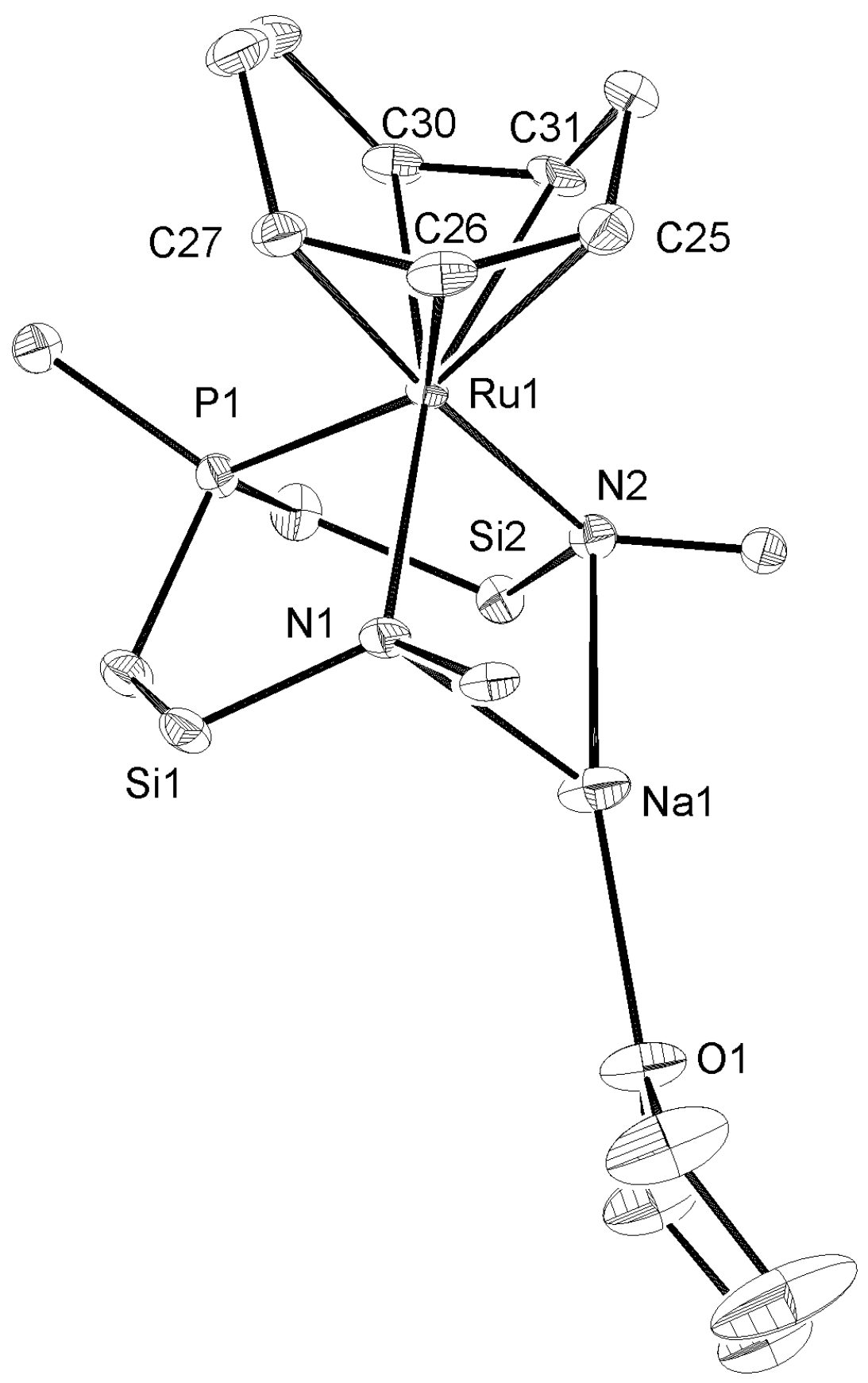

Figure S5. ORTEP representation (50\% thermal ellipsoids) of the solid-state molecular structure of $[\mathrm{Na}(\mathrm{THF})]\left([\mathrm{NPN}] \mathrm{Ru}\left(\eta^{3}: \eta^{2}-\mathrm{C}_{8} \mathrm{H}_{11}\right)\right)(\mathbf{5})$ as determined by X-ray diffraction. The [NPN] silyl methyl groups have been omitted for clarity and only the ipso carbon atoms of the amido and phosphine phenyl rings are shown. 
Table S7. Crystallographic Data and Structure Refinement Data for complex $[\mathrm{Na}(\mathrm{THF})]\left([\mathrm{NPN}] \mathrm{Ru}\left(\eta^{3}: \eta^{2}-\mathrm{C}_{8} \mathrm{H}_{11}\right)\right)(\mathbf{5})$.

\begin{tabular}{|c|c|}
\hline & 5 \\
\hline Formula & $\begin{array}{l}\mathrm{C}_{36} \mathrm{H}_{50} \mathrm{~N}_{2} \mathrm{NaPSi}_{2} \\
\mathrm{RuO} . \mathrm{C}_{7} \mathrm{H}_{8}\end{array}$ \\
\hline Fw & 814.15 \\
\hline Colour, Habit & orange, chip \\
\hline Crystal size, mm & $\begin{array}{llll}0.25 & \mathrm{x} & 0.25 \times \\
0.10 & & & \end{array}$ \\
\hline Crystal system & triclinic \\
\hline Space group & P1- (\#2) \\
\hline $\mathrm{a}, \AA$ & $10.2121(4)$ \\
\hline $\mathrm{b}, \AA$ & $10.3162(3)$ \\
\hline $\mathrm{c}, \AA$ & $22.133(1)$ \\
\hline$\alpha, \operatorname{deg}$ & $87.707(7)$ \\
\hline$\beta$, deg & $79.077(6)$ \\
\hline$\gamma, \operatorname{deg}$ & $64.334(4)$ \\
\hline $\mathrm{V}, \AA^{3}$ & $2061.3(2)$ \\
\hline $\mathrm{Z}$ & 2 \\
\hline $\mathrm{T},{ }^{\circ} \mathrm{C}$ & $-100 \pm 1$ \\
\hline$\rho_{\text {calc }}, \mathrm{g} / \mathrm{cm}^{3}$ & 1.312 \\
\hline $\mathrm{F}_{000}$ & 856.00 \\
\hline$\mu(\operatorname{MoK} \alpha), \mathrm{cm}^{-1}$ & 5.20 \\
\hline correction factors & $0.8093-1.0000$ \\
\hline $2 \theta_{\max }, \operatorname{deg}$ & 55.7 \\
\hline total no. of reflns & 18786 \\
\hline no. of unique reflns & 8409 \\
\hline $\mathrm{R}_{\text {int }}$ & 0.032 \\
\hline $\begin{array}{l}\text { no. observations (I } \\
>\text { n } \sigma(\mathrm{I}) \text { ) }\end{array}$ & $7131(n=3)$ \\
\hline no. of variables & 480 \\
\hline $\mathrm{R}\left(\mathrm{F}^{2}\right.$, all data $)$ & 0.052 \\
\hline $\mathrm{R}_{\mathrm{w}}\left(\mathrm{F}^{2}\right.$, all data $)$ & 0.082 \\
\hline $\mathrm{R}(\mathrm{F}, \mathrm{I}>\mathrm{n} \sigma(\mathrm{I}))$ & $0.029(n=3)$ \\
\hline $\mathrm{R}_{\mathrm{w}}(\mathrm{F}, \mathrm{I}>\mathrm{n \sigma}(\mathrm{I}))$ & $0.037(n=3)$ \\
\hline Gof & 1.48 \\
\hline
\end{tabular}

Rigaku/ADSC CCD diffractometer, $\mathrm{R}=\Sigma|| \mathrm{F}_{\mathrm{o}}^{2}|-| \mathrm{F}_{\mathrm{c}}|| /\left|\mathrm{F}_{\mathrm{o}}\right| \mathrm{R}_{\mathrm{w}}=\left[\Sigma\left(\mathrm{F}_{\mathrm{o}}{ }^{2}-\mathrm{F}_{\mathrm{c}}{ }^{2}\right)^{2} / \Sigma \omega\left(\mathrm{F}_{\mathrm{o}}{ }^{2}\right)^{2}\right]^{1 / 2}$. 


\section{References}

1) $d^{*} T R E K$; Area Detector Software. Version 4.13. Molecular Structure Corporation, 1996-1998.

2)Altomare, A.; Cascarano, M.; Giacovazzo, C.; Guagliara, A. J. Appl. Cryst. 1994, 26, 343.

3)Altomare, A.; Burla, M. C.; Cammalli, G.; Cascarano, M.; Giacovazzo, C.; Guagliardi, A.; Moliterni, A. G. G.; Polidori, G.; Spagna, A. J. Appl. Cryst. 1999, 32, 115-119.

4)Beurskens, P. T.; Admiraal, G.; Beurskens, G.; Bosman, W. P.; Gelder, R. d.; Isreal, R.; Smits, J. M. M. DIRDIF94; The DIRDIF-94 program system, Technical Report of the Crystallography Laboratory: University of Nijmegen, The Netherlands, 1994. 